\title{
Influence of intensity of illumination on weed recognition algorithm
}

\author{
M. A. Mirzaev*, A.I. Dyshekov, and I.G. Smirnov \\ Federal Scientific Agroengineering Center VIM, Moscow, 1st Institutsky passage, 5, Russia
}

\begin{abstract}
This study explores the influence of daylight illumination on operating of autonomous robotic weed control complex. The illumination value is taken from minimum on the order of a few lux (high overcast) to maximum exceeding one hundred twenty thousand lux (bright noon). This was done to cover the entire daylight illumination range. This experiment is not focused on operating of the algorithm using the artificial light sources since they are supposed to be easily adjusted with the proper equipment. The performance of the algorithm at various illumination levels is evaluated by the surface area of the recognized beet crops on early stages of growth.
\end{abstract}

\section{Introduction}

One of the aims of sustainable agriculture is to boost crop yield while reducing dependence on herbicides and pesticides to control weed growth in particular. There are different methods of weed control including remote sensing, spectral remission and machine vision [1, 2]. Remote sensing is restricted by spatial and temporal resolutions [3]. Spectral remission is based on the levels of weed infestation in the probing zone. It makes this method insufficient to detect a single weed due to low spatial resolution in addition to considerably higher cost of these devices. Machine vision demonstrates the highest potential for overcoming the restrictions of other systems due to high spatial resolution and relatively low cost of equipment.

In this study machine vision is employed to recognize weed vegetation using the method of image segmentation and recognizing the crop [4, 5].

The main disadvantage of this method is dependance on the change of illumination level. The unstable illumination can be caused by cloud cover, change concerning position of the sun, shadows cast by large agricultural machinery. These factors reduce the amount of light falling on the cultivated soil and, therefore, the quality of the received image. The most serious potential impediment caused by the change of illumination level is low processing of the image, which can be minimized by automatic adjustment to the environmental conditions of shutter speed or aperture. Furthermore the change of illumination level and shadowing may result in low efficiency or unsuitability of traditional methods of processing segmented images [6].

\footnotetext{
* Corresponding author: mirza.pochta@gmail.com
} 
The purpose of this study is to evaluate operating conditions of the recognition algorithm of autonomous robotic weed control complex during the daytime with various illumination levels.

\section{Materials and methods}

The experiment was conducted on a laboratory bench simulating the working environment of the autonomous robotic complex for pinpoint weed control. The bench consists of a platform with guide rails and a carriage on it which is driven by stepper motors within the constraints. A video camera and an operating device, which turns on the system of simultaneous mechanical and chemical weed removal, connected to a herbicide tank through a pump are installed on the carriage. A control unit and an electronic computer with specialized software for image processing $[7,8]$ are located beyond the working area.

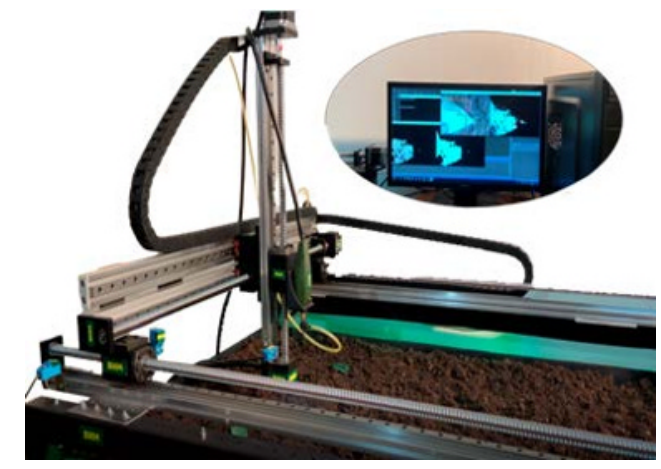

Fig. 1. The autonomous robotic weed control complex developed during the research work.

The figure above shows a work unit developed on the basis of the Federal Scientific Agroengineering Center of the Federal State Budgetary Scientific Institution VIM.

A photo of the soil sample for the study is shown in Figure 2.

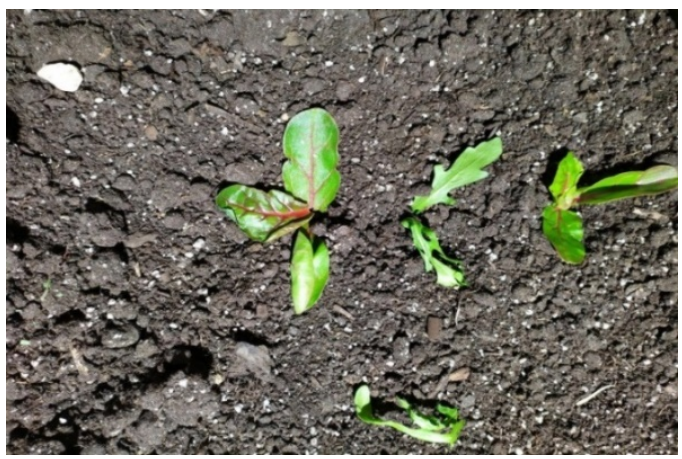

Fig. 2. Photograph of a soil sample for the research

By the method of illumination, computer vision systems can be divided into passive processing the images obtained under natural lighting conditions, and active - using a certain radiation generator (for example, radar systems, laser rangefinders, systems with structured lighting, etc.) [9].

As already mentioned, the images vary significantly. Changing the shooting angle, illumination, mutual displacing of the objects and their parts with their partial obstruction result in complex transformations in the pixel brightness with constant image content. This 
is called image variability. As a result, single image dots and even rather large image areas may be insufficient to solve vision tasks. For example, the color of the particular object significantly depends on the illumination. A white sheet of paper in dim light will be less bright than a dark gray sheet in bright light.

Modern components of machine vision illumination for industrial use consist of:

- light sources

- mechanical adjustment elements

- $\quad$ fiber optical elements

- $\quad$ stabilizing, controlling and interface electronics

- $\quad$ software [10].

This study examined the influence of the natural illumination throughout the daytime since it cannot be adjusted to the existing equipment, whereas at night it is possible to resort to the aforementioned technical systems, which allow adjusting of the required lighting parameters.

The daylight is a set of direct and indirect sunlight throughout the daytime which comprises of directional light of sun rays, diffused light from the cloudless sky and the light scattered by the clouds. Thus, daylight excludes moonlight, despite it being indirect sunlight.

Illumination values of daylight can vary from 120000 lux for direct sunlight st noon (which can cause eye pain) to less than 5 lux in case of thick storm clouds with the sun on the horizon.

An uninterrupted spectrum is the electromagnetic spectrum, in which the energy distribution is characterized by continuous function of wavelength of illuminating light $f(\lambda)$ (or its frequency $\varphi(v)$ ). For the continuous spectrum function $f(\lambda)$ slightly varies over a rather wide range of wavelength, unlike line and band spectra, when $f(\lambda)$ has pronounced maxima for discrete values of the wavelength $\lambda$, that are very narrow for spectral lines and wider for spectral stripes. In the visible range dispersion of light with spectral instruments gives continuous spectrum in a form of uninterrupted colored stripe (during visual observation) or a smooth curve (during photoelectric recording). An equilibrium spectrum is an example of the continuous spectrum covering the entire frequency range, characterized by certain spectral energy distribution. It is characterized by Planck distribution law.

The following is the spectrum of widely used sources of light.

The figure 3 shows a range of commonly used light sources.
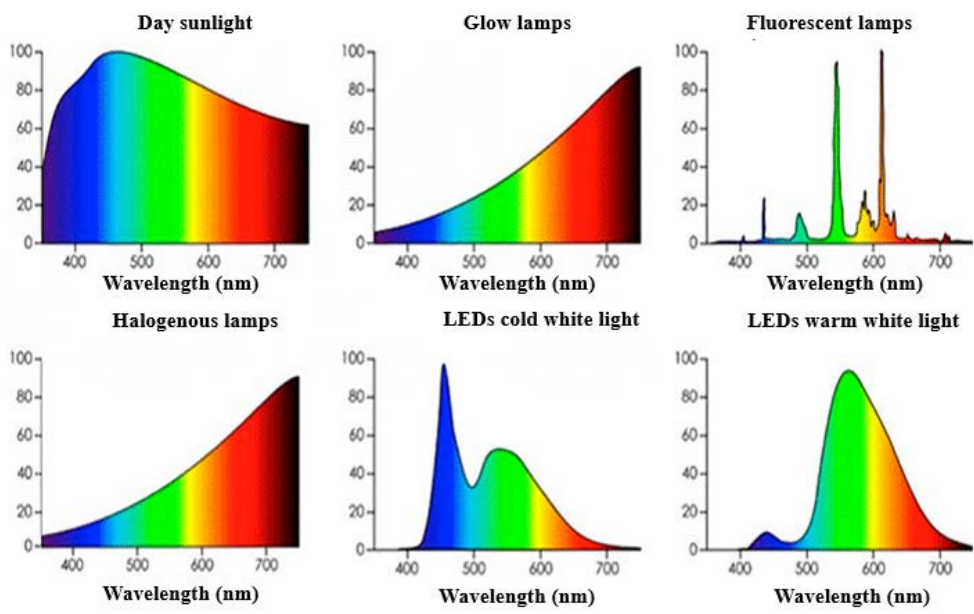

Fig. 3. Spectrum of different light sources 
In the course of the study the following indicators were reviewed.

CQS (Color quality scale) is a new lighting source quality parameter developed by NIST (National Institute of Standards and Technology) for the new solid-state lighting markets. CQS uses a richer color and proposed 15 new colors to evaluate the light sources, a value of 100 represents the best quality of the light source, 0 represents the worst quality of the light source.

$\mathrm{CRI}(\mathrm{Ra})$ (color rendering index) is an average value of $\mathrm{R} 1 \sim \mathrm{R} 8$. A value of 100 indicates the best light source quality, whereas 0 indicates the worst light source quality.

\section{Results and discussion}

The illumination changes significantly throughout a daytime. During the night it is possible to adjust proper light spectrum without any difficulties for the optimal implementation of the activities of epy weed control using machine vision. There is no such possibility regarding daytime and in spite of the apparent simplicity of this task it is difficult to customize an algorithm. One of such problems is extremely high solar activity which entrails incorrect color rendering, light exposure and other negative effects which, in turn, result in increasing number of mistakes. Furthermore, considering the fact that sunlight differs from artificial lighting due to the wide distributed wavelength spectrum, we also decided to regard minimum illumination values $[11,12]$.

The first measurement showed minimum value of 8 lux. Figure 4 shows the results of spectrum analysis and spectral content quality scales CRI and CQS.

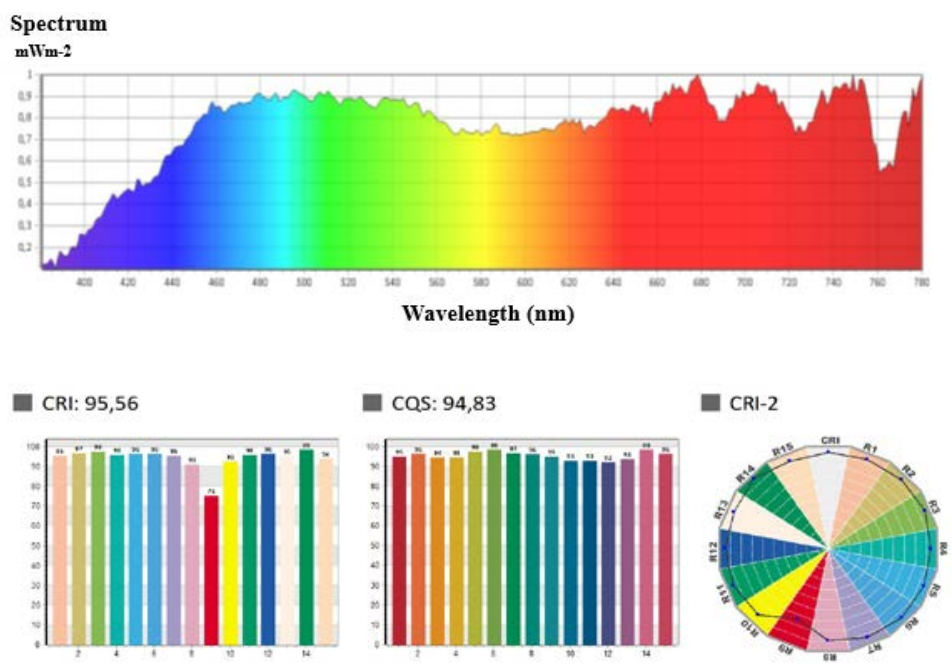

Fig. 4. The results of the first lighting parameters measurement. 


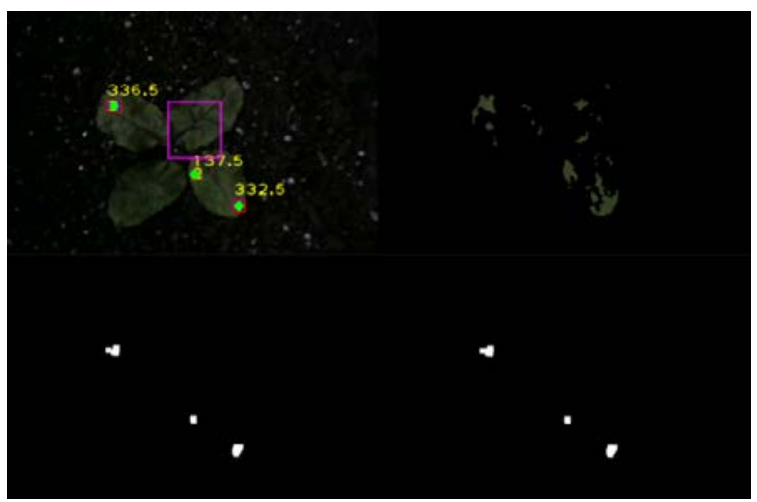

Fig. 5. The first measurement output of the recognition algorithm.

Figures 5 and 6 represent the photos of recognized images of soil for the first and second experiments.

Subsequently the lighting was gradually increased. The second measurement shows the value of around 500 lux. The output of the algorithm is showed on the figure 6 .

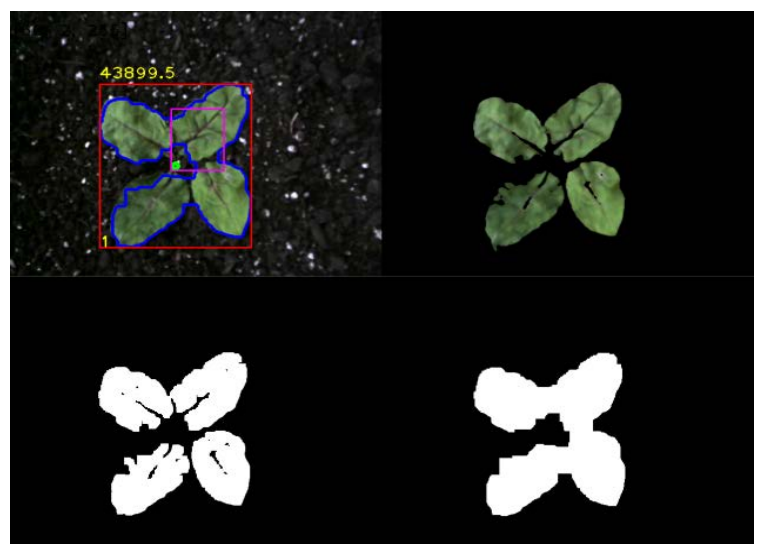

Fig. 6. The results of the second experiment.

The further measurements are showed in the table below.

Table 1. Experimental values

\begin{tabular}{|r|r|r|r|r|}
\hline № & \multicolumn{1}{|c|}{ CRI, \% } & $\begin{array}{c}\text { CQS, } \\
\text { \% }\end{array}$ & \multicolumn{1}{c|}{ Illumination, $\mathbf{~ x ~}$} & \multicolumn{1}{c|}{ Area, pixels } \\
\hline 1 & 95,56 & 94,83 & 8 & 335,5 \\
\hline 2 & 96,68 & 94,96 & 20 & 43899,5 \\
\hline 3 & 96,83 & 95,24 & 500 & 46844 \\
\hline 4 & 97,08 & 96,03 & 1000 & 50323,5 \\
\hline 5 & 98,29 & 97,43 & 5000 & 51891 \\
\hline 6 & 98,31 & 98,44 & 13000 & 52529 \\
\hline 7 & 98,23 & 98,19 & 20000 & 52703,5 \\
\hline 8 & 98,24 & 98,39 & 41000 & 52593,5 \\
\hline 9 & 98,43 & 98,29 & 58000 & 51597 \\
\hline 10 & 98,56 & 98,75 & 81000 & 50425,5 \\
\hline 11 & 98,82 & 98,99 & 97000 & 49115,5 \\
\hline 12 & 96,64 & 97,01 & 127000 & 48585 \\
\hline
\end{tabular}




\section{Conclusions}

Figure 7 shows a graph for evaluating a light source using a light quality scale developed by NIST (National Institute of Standards and Technology) for new solid-state lighting markets. The value of one hundred percent represents the best lighting source quality, zero represents the worst lighting source quality. In this case, high light quality is obvious due to high rates approaching one hundred percent in course of experiments. The lowest rate corresponds to $94 \%$ with minimum illumination. It is a result of stronger influence of extraneous light sources due to lower illumination source simulating solar radiation. The average rate is 98 per cent.

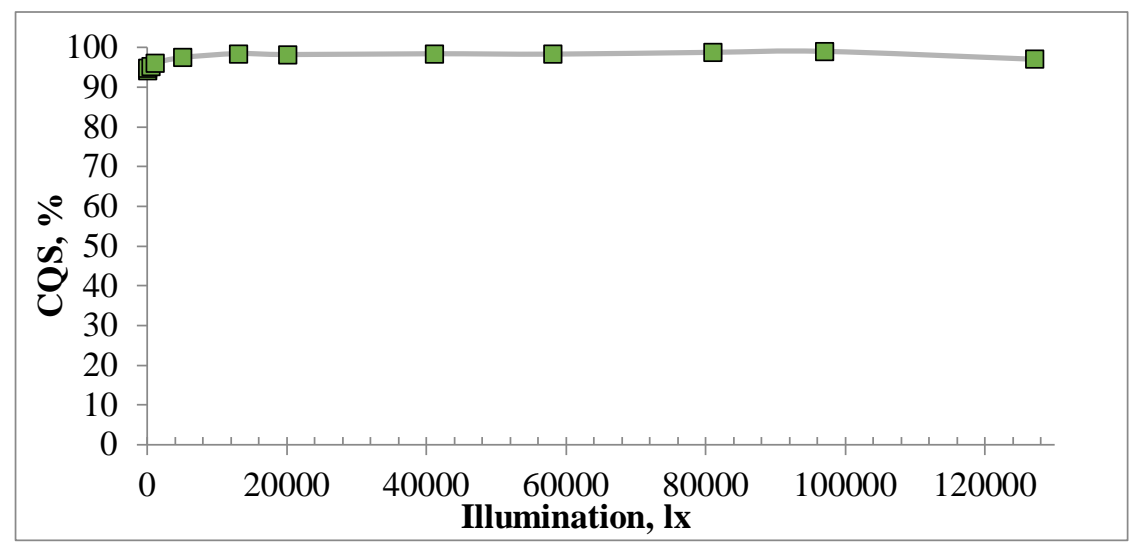

Fig. 7. CQS value diagram for the experiments

Figure 8 shows a graph for evaluating the color rendering index of a light source. The value of 100 indicates the best light source quality, whereas the value of 0 represents the worst quality similar to the indicator above. Minimal value of $96 \%$ in midrange exceeds $98 \%$.

Both diagrams show a slight decrease of light source characteristics in the last experiment describing the maximum illumination. This is possible due to the increase in the amount of reflected light during the experiment.

In total it can be considered that the illumination in each experiment corresponds to the operating conditions of the autonomous robotic weed control complex.

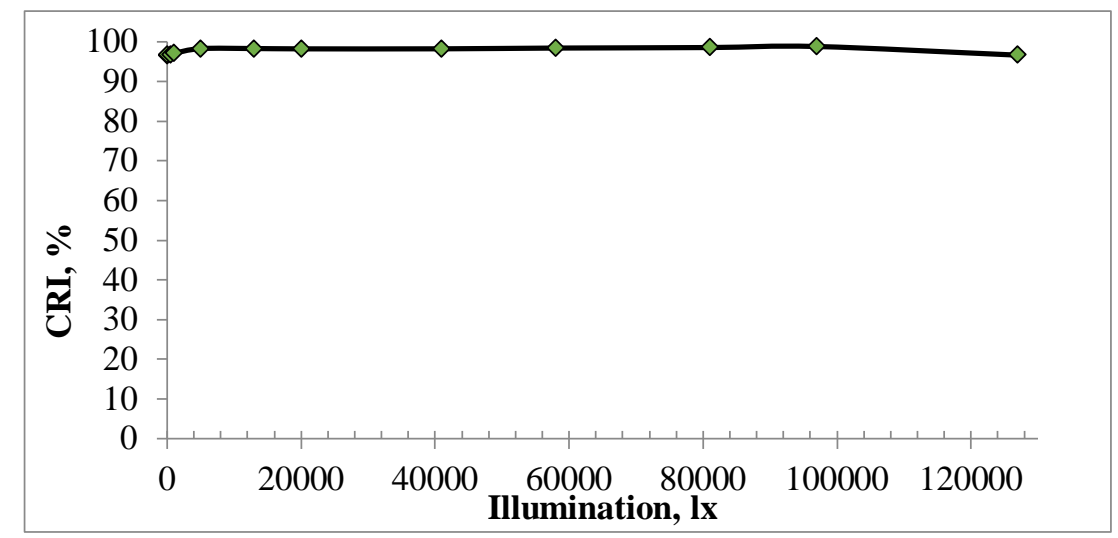

Fig. 8. The diagram of CRI values for the experiments 
Figure 9 shows the dependence of recognized surface on the illumination with natural type light source. The same plant for all illumination values was taken to evaluate the performance of the algorithm. The other parameters such as the height of the lens above the soil level, the same operating mode of the recording device, etc. were also constant. The distributed surface is directly proportional to the number of pixels with the recognized algorithm.

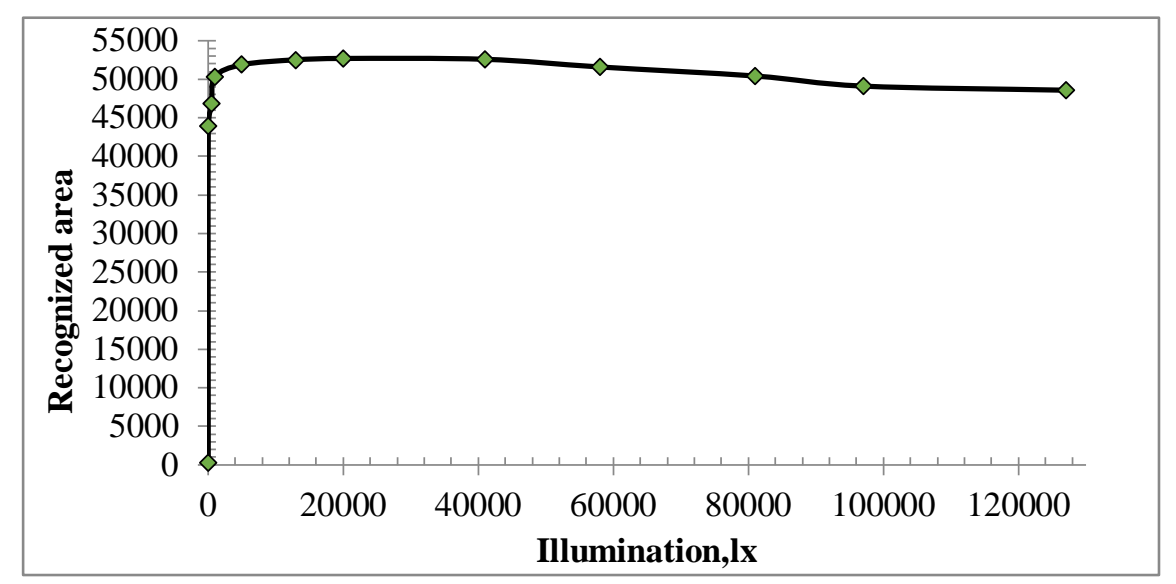

Fig. 9. The area of the recognized contour on the image taken with the lens $20 \mathrm{~cm}$ above the soil level

The following results were obtained from the experiment. The algorithm was tested at the minimum illumination value of 8 lux corresponding to extremely low light. This experiment shows unacceptably poor performance. In this case the optimum solution is to use artificial light sources since the influence natural light is insignificant.

The next relatively insignificant increase of illumination allowed the algorithm to recognize the crop of sugar beet in high quality. This experiment was conducted at a value of 20 lux. The continuing increase of illumination intensity improved the recognition quality.

The maximum quality was obtained in the range from 5000 to 60000 lux. This range extends throughout the daytime.

With the further illumination increase the quality of recognition has slightly decreased. The decrease was caused by the excessive illumination which affected the performance of the recording device. In conditions of maximum illumination the algorithm still adequately identified the plants.

The artificial light sources which induce analogous to natural light in the average range of experiments can be used to provide the night work of the algorithm.

In conclusion, the recognition algorithm of the robotic weed control complex is applicable in the field throughout 24-hour period.

\section{References}

1. J. L. Hatfield, A.A. Gitelson, J.S. Schepers, C.L Walthall, Application of spectral remote sensing for agronomic decisions, Agronomy Journal, vol. 100, pp. 117-131 (2008)

2. D. Show, F. Lopez, M. Jurado-Expósito, Translation of Remote Sensing Data into Weed Management Decisions, Weed Science, v. 53, No.2, pp. 264-273 (2005)

3. L.F. Tian, A Review on Remote Sensing of Weeds in Agriculture, Precision Agriculture, v. 5, pages 477-508 (2004)

4. A.I. Dyshekov, I.G. Smirnov, M.A. Mirzaev, M.A. Shereuzhev, Published Principles 
of functioning of the autonomous device for weed control for precision agriculture, Published under licence by IOP (2020)

5. N. Kozlov, Image segmentation and transformations preserving the shape of figures Intilligentnie sistemy. Teoriya i practica, v. 23, No. 2. pp. 57-70 (2019) (in Russian)

6. E.E. Gazieva, A.V. Vasiliev, V.I Syriamkin, V.I. Yurchenko Lighting systems in vision equipment for monitoring electronic devices and systems, Tezisy docladov 8 naychnotehnicheskoy konferenzii «Electronie i electromehanicheskie sistemy I ystroistva», pp. 339-340 (2010) (in Russian)

7. J.B. Gerrish, T.C. Surbrook, Mobile Robots in Agriculture, Robotics and intelligent machines in agriculture: proceedings of the first International Conference on Robotics and Intelligent Machines in Agriculture, October 2-4, American Society of Agricultural Engineers, pp. 30-41 (1983) (in Russian)

8. L. Cooke, Smart sprayer selects weeds for elimination, Agricultural Research, v. 44(4) (1996)

9. D.E. Peterson, Weed Detection Using Color Machine Vision (Transactions of the ASAE, Blasko, 1978)

10. S.D. Noble, R.B. Brown, T.G. Crowe, The Use of Spectral Properties for Weed Detection and Identification - A Review (AIC meeting Saskatoon, Saskatchewan, 2002)

11. J. Hemming, T. Rath, PA-Precision Agriculture: Computer-Vision-based Weed Identification under Field Conditions using Controlled Lighting Journal Agric. Eng v. 78, pp. 233-243 (2001)

12. D.E. Guyer, G.E. Miles, M.M. Schreiber, O.R. Mitchell, V.C. Vanderbilt, Machine vision and image processing for plant identification Transactions of the ASAE, v. 29 (6), pp. 1500-1507 